\title{
A Novel Non-destructive Interfacing Technique for Molecular Scale Switching Junctions
}

Chad Johns ${ }^{1}$, Ibrahim Kimukin ${ }^{1}$, M. Saif Islam ${ }^{1}$, Doug A. A. Ohlberg ${ }^{2}$, Carrie Donley ${ }^{2}$, Duncan R. Stewart ${ }^{2}$, Shih-Yuan Wang ${ }^{2}$, and R. Stanley Williams ${ }^{2}$

${ }^{1}$ Electrical Engineering, University of California, Davis, One Shields Ave., Davis, CA, 95616

${ }^{2}$ Quantum Science Research, Hewllet Packard, 1501 Page Mill Road, Palo Alto, CA, 94304

\section{ABSTRACT}

Characterization of molecular scale electronic devices generally involves deposition of a metal top electrode onto an organic film. During the evaporation process, high-energy granules of metals may lead to unwanted reactions between the organic molecules and deposited top electrodes. This can cause, as commonly reported, lasting damage which leaves most junctions either shorted or an open circuit. To overcome this important issue of physical damage to the molecules, we developed a novel technique of interfacing molecules by laying a prefabricated metallic electrode on top of a monolayer of molecules which were deposited onto another set of electrodes. A monolayer of cadmium stearate $\left(\left(\mathrm{C}_{17} \mathrm{H}_{35} \mathrm{COO}\right)_{2} \mathrm{Cd}\right)$ was deposited, using the Langmuir-Blodgett technique, onto platinum (Pt) electrodes which further rested on 200nm of oxide used for isolation. A separate set of aluminum with native oxide $\left(\mathrm{Al} / \mathrm{AlO}{ }_{\mathrm{x}}\right)$ electrodes were fabricated on a different oxide free, highly p-doped substrate and was gently placed on the cadmium stearate monolayer. Electrical connection to the top electrode was accomplished by probing the backside of the highly doped wafer whereas the bottom electrodes were individually addressed. Pressure was applied to ensure firm contact between the molecules and the top electrodes. Preliminary results showed an observed switching voltage of $3 \mathrm{~V}-4 \mathrm{~V}$. Most of the devices with $\mathrm{Al}$ as the top electrodes exhibited a gradual progression from switching on the positive side, closing or no longer switching, and then switching towards the negative side.

\section{INTRODUCTION}

Molecular electronics can be loosely defined as a branch of nanotechnology that utilizes single molecules or small groups of molecules as components in electronic devices. It holds the promise of allowing the next generation of device scaling into the nanometer range. Currently, the synthesis, characterization and device implementation of new molecular compounds is a very active research area. The majority of the testing and characterization of new molecules is done with an AFM/STM package or break junctions ${ }^{1}$. With the ultimate goal being that of a device, this type of process is impractical since each junction would require a separate AFM/STM tip or break junctions. The alternative that many groups have adopted is using some form of a cross junction in which the monolayer of molecules is sandwiched between two metallic electrodes ${ }^{2}$. Although this is very practical for fabrication of various logic circuits, a new problem arises from the physical vapor deposition of the top electrode, particularly that of low yields. Energetic clusters or atoms from the evaporation or sputtering of the top electrode can cause irreversible damage to the monolayer of molecules. If the organic monolayer is not directly damaged from the energetic clusters, then there is also the possibility of unwanted reactions driven by the added energy, as been reported for titanium and chromium ${ }^{3}$. Lastly, there is the possibility of a metal filament penetrating through the monolayer, leaving the junction shorted. To overcome this 
important issue of physical damage to the molecules, we developed a novel technique of interfacing molecules by laying prefabricated metallic electrodes on top of a monolayer of molecules.

\section{EXPERIMENT}

The bottom electrode started with 200nm of thermally grown $\mathrm{SiO}_{2}$ on silicon in order to electrically isolate several metal electrode lines. Platinum electrodes were then lithographically defined with critical dimensions of $1 \mu \mathrm{m}$. A monolayer $(\sim 2.8 \mathrm{~nm})$ of cadmium stearate $\left(\left(\mathrm{C}_{17} \mathrm{H}_{35} \mathrm{COO}\right)_{2} \mathrm{Cd}\right)$ was deposited using the Langmuir-Blodgett technique. As seen in Figure 1, a separate prefabricated top electrode consisted of lithographically defined aluminum electrodes with a thin layer of native oxide, similar to experiments done by Richter et al. ${ }^{4}$, on a highly doped p-type wafer. The cross junction area was $1 \mu \mathrm{m} \times 1 \mu \mathrm{m}$. The top electrode piece was carefully placed onto the bottom electrode piece containing the monolayer. The $1 \mu \mathrm{m}$ metal lines of the bottom piece were individually probed and addressed while electrical connection to the top electrode was done by probing the back-side of the highly doped wafer. Voltage was swept in a loop fashion (ex. $0 \mathrm{~V}$ to $4 \mathrm{~V}$ to $-4 \mathrm{~V}$ then back to $0 \mathrm{~V}$ ) while current was measured. A control sample, without the monolayer of cadmium stearate exhibited very low resistance and no hystersis.

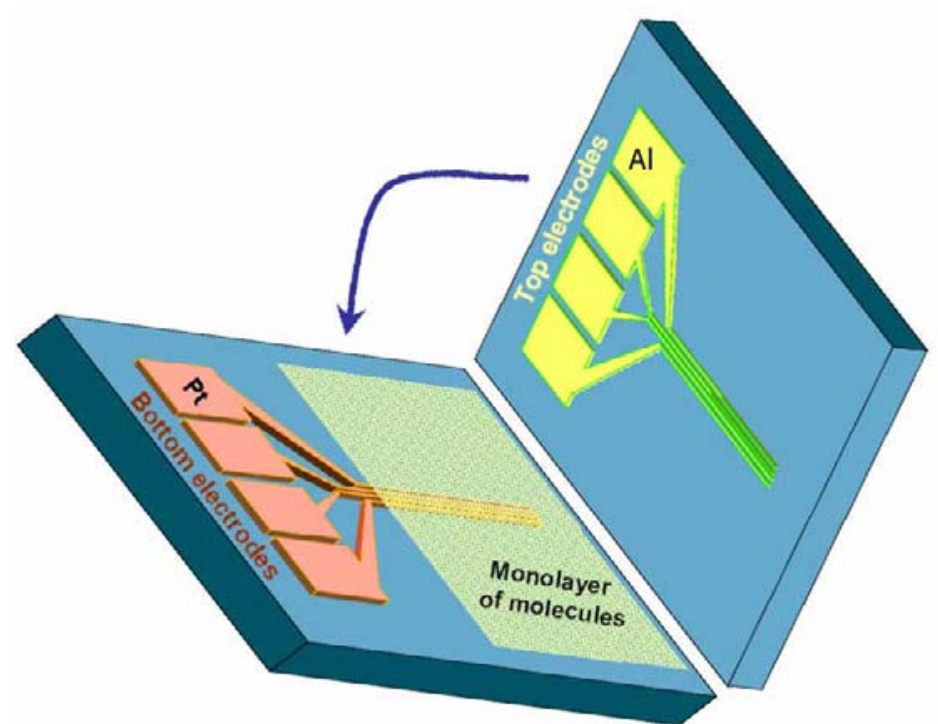

Figure 1: Two separate prefabricated electrodes were brought together, thus avoiding the damage and problems of the monolayer associated with the deposition of the top electrode.

\section{DISCUSSION}

For a first demonstration, the average yield was $\sim 30 \%$. We believe the yield can be further improved by conducting the experiment in more controlled environment. Our experiment was conducted in a regular, non-cleanroom setting and we observed that issues such as dust trapped between the electrodes could easily make a whole section of electrodes nonfunctional by introducing a gap between electrodes. Also, the roughness of the electrodes could easily cause unwanted shorts. It has been recently shown that yields can be dramatically increased through 
the use of chemical mechanical polishing of the metal electrodes ${ }^{5}$. This is a further possibility for the future. As a first demonstration, such issues were not addressed. As seen by the representative curves in Figure 2, switching, similar to published results ${ }^{6}$, between an on (high) state and off (low) state was observed. The first cycle was the lowest curve on the graph.

Subsequent voltage cycles can be found directly above offset by $15 \mu \mathrm{A}$. The sample finished in its high state (shown by the highest curve) in which switching no longer occurred. As indicated by the arrows, the sample switched from a low state to that of a high or conduction state.

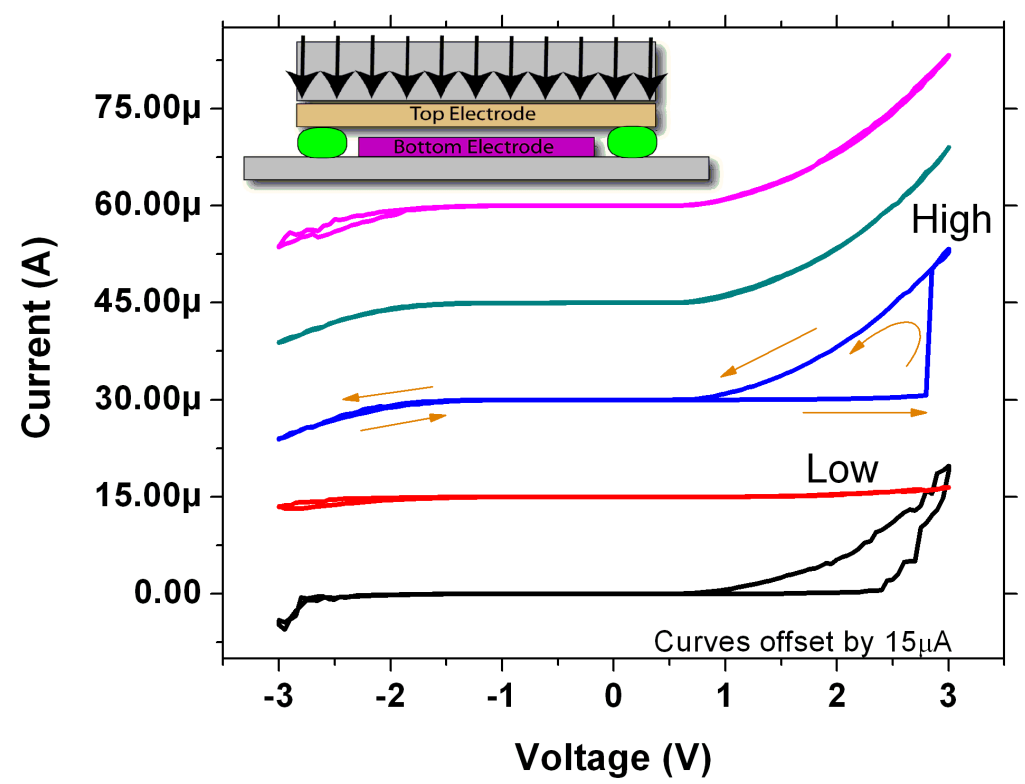

Figure 2: Switching between high and low states.

Experiments concerning the effects of pressure on the switching behavior were performed. Current-voltage measurements were taken after the two electrodes were brought together. Curves 1 and 2 in Figure 3 depict very low current at a low pressure $\mathrm{P}_{1}$. As higher pressure $\left(\mathrm{P}_{2}\right)$ was applied, an increase in the current was observed (curves 3 and 4), most likely due to a reduction of the tunneling gap as similarly seen by Tivanski et al ${ }^{7}$. At this pressure it is believed the contact or bonding between the electrode and molecule was poor and conduction was mainly due to tunneling. 


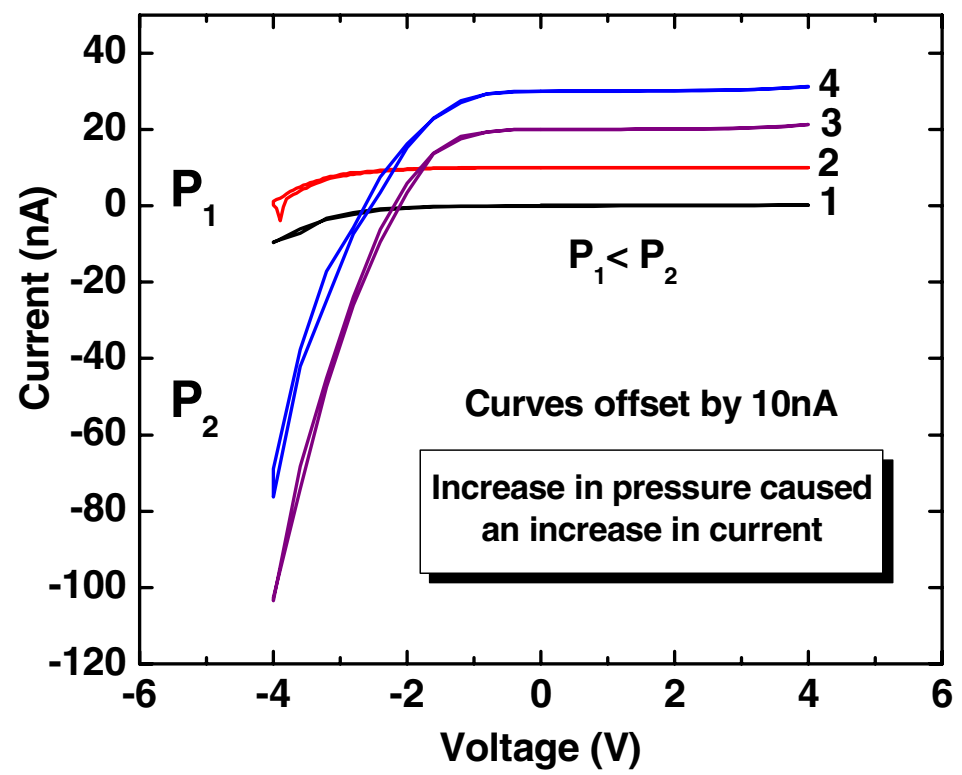

Figure 3: An increase in pressure caused an increase in observed current due to the reduction of the tunneling barrier.

When the pressure was further increased, the current increased by three orders of magnitude from nanoamps to microamps as shown in Figure 4. The sample also began to switch from a low to high state similar to other samples (Figure 2). Our current density at $1 \mathrm{~V}(1 \mu \mathrm{m} \mathrm{x}$ $1 \mu \mathrm{m}$ ) of about $5 \times 10^{6} \mathrm{~A} / \mathrm{m}^{2}$ is an order of magnitude higher than what was observed by Kuekes et al. who used the sample monolayer of cadmium stearate between two platinum electrodes ${ }^{8}$. In their experiment cross junction of $12 \mu \mathrm{m}^{2}$ and $16 \mu \mathrm{m}^{2}$ were used. At this pressure, the top electrodes probably made good contact with the monolayer of cadmium stearate allowing switching to occur. Although consistent switching was observed, the sample changed back to nanoamps after a few cycles as seen in the inset of Figure 4. The sample was then believed to have failed or the contact between the electrode and the molecule was lost. The majority of the samples tested failed in this manner where the current decreased until an open circuit was observed. We are currently working on correlating the applied pressure with the magnitude of observed current and switching behavior. 


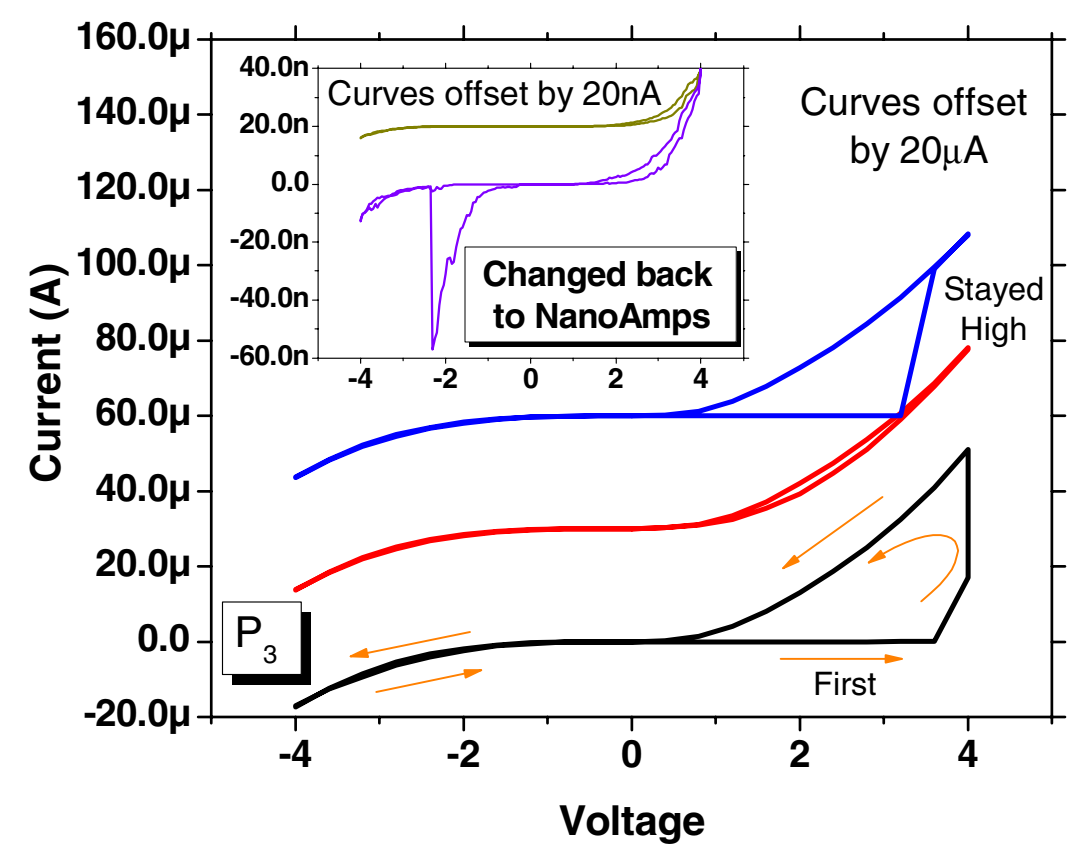

Figure 4: As pressure was further increased, the current jumped 1000 fold and started switching consistently. After several cycles, the current dropped back to nanoamps.

After the devices were found to fail by showing properties similar to open circuits, we found several of them to suddenly show switching behavior. Figure 5 depicts this unexpected switching phenomenon with a peak on-off current ratio of $2.5 \times 10^{4}$. Also noticed in several samples, and that of Figure 5, was a gradual progression of one side of the switching loop closing while the opposite side opened up. The cause of this observed phenomenon is currently under investigation.

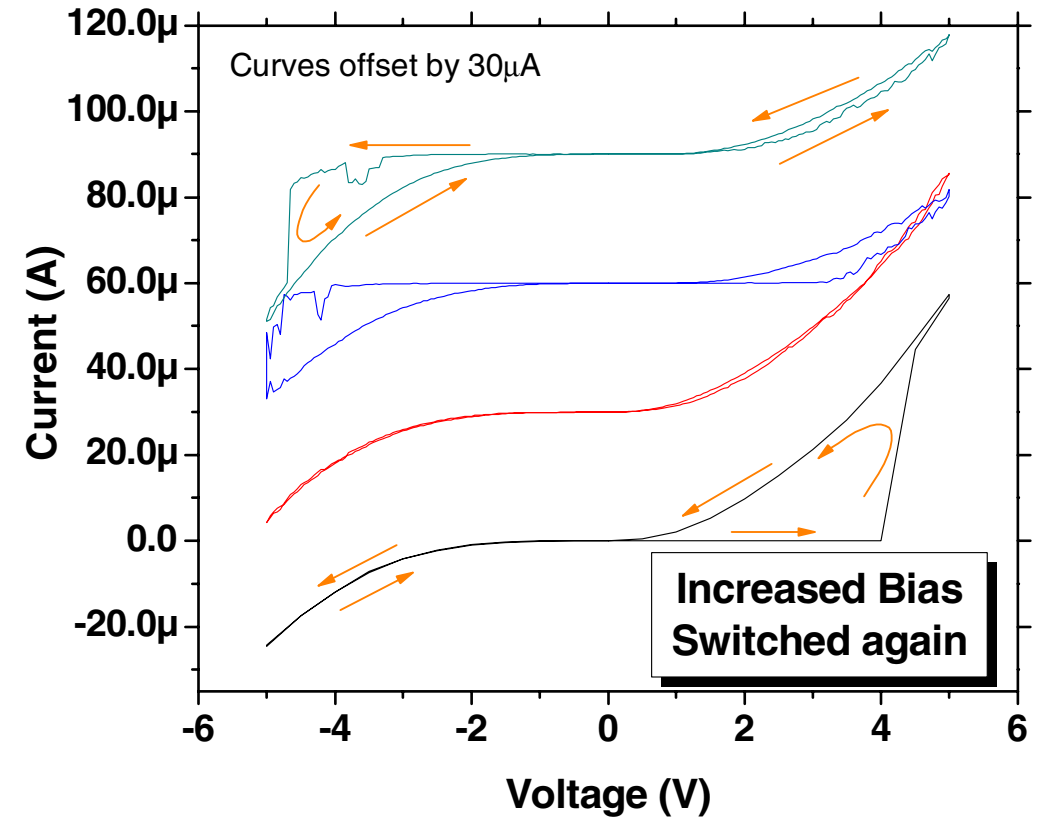

Figure 5: Bias was increased to a 5V sweep and switching was observed again. A gradual progression of the positive switching loop closing while the negative loop opening was observed. 


\section{CONCLUSIONS (OR RESULTS)}

A simple, fast, and inexpensive non-destructive interfacing technique was demonstrated by laying prefabricated metallic electrodes on top of a monolayer of molecules. Excellent results were obtained using a top electrode of $\mathrm{Al} / \mathrm{AlO}_{\mathrm{x}}$ placed on top of a Pt electrode with an $\mathrm{LB}$ film of cadmium stearate. A switching voltage of around $3 \mathrm{~V}$ to $4 \mathrm{~V}$ was observed with an on-off current ratio of $\sim 10^{4}$. These preliminary results also showed a yield of around $30 \%$ which can be improved in the future with well controlled processing. The magnitude of the measured current was found to depend on the amount of applied pressure, consistent with a model where the tunneling barrier decreases with an increase in pressure. This powerful technique can be used to interface large arrays of switching junctions and as a test-vehicle for accurate characterization of the molecular properties without causing any damage during the device fabrication process.

\section{REFERENCES}

1 M. A. Reed, C. Zhou, C. J. Muller et al., "Conductance of a molecular junction," Science 278 (5336), 252-254 (1997); M. T. Cygan, T. D. Dunbar, J. J. Arnold et al., "Insertion, conductivity, and structures of conjugated organic oligomers in self-assembled alkanethiol monolayers on $\mathrm{Au}\{111\}$," Journal of the American Chemical Society 120 (12), 2721-2732 (1998).

2 J. Chen, W. Wang, M. A. Reed et al., "Room-temperature negative differential resistance in nanoscale molecular junctions," Applied Physics Letters 77 (8), 1224-1226 (2000); Y. Chen, D. A. A. Ohlberg, X. M. Li et al., "Nanoscale molecular-switch devices fabricated by imprint lithography," Applied Physics Letters 82 (10), 1610-1612 (2003). G. C. Herdt, D. R. Jung, and A. W. Czanderna, "Penetration of deposited Ag and Cu overlayers through alkanethiol self-assembled monolayers on gold," Journal of Adhesion 60 (1-4), 197-222 (1997).

4 C. A. Richter, D. R. Stewart, D. A. A. Ohlberg et al., "Electrical characterization of Al/AlOx/molecule/Ti/Al devices," Applied Physics a-Materials Science \& Processing 80 (6), 1355-1362 (2005).

5 M. S. Islam, G. Y. Jung, T. Ha et al., "Ultra-smooth platinum surfaces for nanoscale devices fabricated using chemical mechanical polishing," Applied Physics a-Materials Science \& Processing 80 (6), 1385-1389 (2005). Y. Chen, G. Y. Jung, D. A. A. Ohlberg et al., "Nanoscale molecular-switch crossbar circuits," Nanotechnology 14 (4), 462-468 (2003).

7 A. V. Tivanski, J. E. Bemis, B. B. Akhremitchev et al., "Adhesion forces in conducting probe atomic force microscopy," Langmuir 19 (6), 1929-1934 (2003).

8 P. J. Kuekes, D. R. Stewart, and R. S. Williams, "The crossbar latch: Logic value storage, restoration, and inversion in crossbar circuits," Journal of Applied Physics 97 (3), (2005). 